\title{
Historical Perspective of Accelerometer Technologies
}

\author{
Torben R. LICHT \\ Patrick R. SCHEEPER \\ A/S Briel \& Kjaer \\ DK-2850 Naerum \\ Denmark
}

\section{Introduction}

Basic physical discoveries have in most cases led to useful applications some decades later.

Apart from optical methods making a direct measurement of the kinematic quantity of acceleration possible, the most fundamental physical principle is Newton's second law (1687) relating acceleration to force via a constant of proportionality: The inertial mass.

For measurement of vibration the single degree of freedom system consisting of a mass suspended by a spring plays a crucial role, together with additional physical mechanisms converting (i.e. transducing) the relative motion or force into a directly measurable (mostly electrical) signal.

The most important discoveries for vibration measurements are capacitance (Leyden jar 1745), induction (1819 H.C. Oersted ), strain-gauge (1856 Lord Kelvin), piezoelectricity (1880 P. \& J. Curie), semiconductor components (transistor 1948 W.B.Shockley), piezoresistivity of semiconductors (1954 C.S.Smith) and electron tunnelling (1983 Binnig).

In parallel to the basic physical principles the technologies have advanced with high speed.

In the period 1920 to 1940 vibration measurements started using inductive, capacitive, strain-gauge and piezoelectric principles. An early accelerometer using a Rochelle salt plate in bending mode was designed by P.V. Bruiel in 1943, and manufactured by Brüel \& Kjaer afterwards.

The lead titanate zirconate family of piezoelectric materials were found in the forties, and accelerometers using these materials were marketed in the fifties. Construction and material refinements have made these transducers the most widely used general purpose transducers over the last three decades and still today.

The semiconductor strain-gauges or piezoresistive elements came into use as replacements for the classic metal s- train-gauges in the last half of the fifties and one of the first micromechanical constructions was a pressure sensor using a silicon diaphragm with diffused resistors.

Since then thousands of papers have been published on what today is called micromechanical sensors, mostly based on silicon as material.

One of the first micromechanical accelerometers was also based on piezoresistivity and a bending beam. This was published by Roylance and Angell in $1979^{\prime \prime}$ and many similar constructions have been investigated in the meantime.

Through the eighties detection and construction principles were proposed and investigated, but the number of commercially available sensors is still very small. Most of these are piezoresistive or capacitive.

The main reason for the small number of commercially available sensors is the very fragmented nature of the market. A large number of small companies are making a huge variety of transducers specialised for each their application. To make micromachined sensors require large investments which can only be paid back if the number of transducers sold is sufficiently large or the prices can be put at a very high level.

Therefore the trend has been transducers for the automotive market, e.g. airbag sensors where the potentially low cost batch fabrication can be exploited, and transducers for very specialised applications, e.g. microgravity measurements where price is unimportant.

To understand the new challenges for the designer of accelerometers it is important to know what happens to various parameters when dimensions are scaled.

\section{Scaling}

Most classic accelerometers of today have volumes in the order of $1 \mathrm{~cm}^{3}$, seismic masses of 1 to 30 grams and mounted resonance frequencies of 10 to $30 \mathrm{kHz}$. 
To make feasible constructions in silicon wafers these dimensions $L$ have to be reduced by a factor of about 10 . The resonance should preferably remain constant.

For the mass-spring system this implies the following scaling properties ( $\propto$ denotes proportionality, $F$ the force on the seismic mass due to the acceleration $a$ of the system, $k$ spring stiffness, $M$ seismic mass and $A$ and $t$ cross section and thickness of a simple disc-shaped spring):

$\begin{array}{lll}\text { Dimensions } & \propto & L \\ \text { Seismic mass } & \propto & L^{3}\end{array}$

The resonance frequency is given by

$$
\omega_{0}=\sqrt{\frac{k}{M}} \ldots \ldots \ldots
$$

and the deformation of the spring is given by the formula (Hooke's and Newton's II law)

$$
k x=F=M a \ldots \ldots(2)
$$

To maintain the resonance frequency $\omega_{0}$ constant, $k$ must be proportional to $M$ and therefore

$$
k \propto \frac{A}{t} \propto M \propto L^{3} \ldots \ldots \ldots . . .
$$

(For a bender construction with length, width and thickness $l, w, t$ the expression $A / t$ should be substituted by $w t^{3} / l^{3}$ )

For a constant vibration input signal this system will then see an input force

$$
F \propto M
$$

l.e. the sensitivity for force sensitive transduction principles at $\omega \ll \omega_{0}$ follows

$$
S_{f} \propto M \propto L^{3}
$$

The displacement is

$$
x=\frac{M}{k} a=\frac{a}{\omega_{0}^{2}}
$$

which means that the displacement sensitive device keep its sensitivity constant when $M$ is changed, provided $\omega_{0}$ is kept constant, or

$$
S_{x}=\text { Const. - . - . - (7) }
$$

This also implies that the spring dimensions must change considerably to fulfil

$$
\frac{A}{t} \propto L^{3} \ldots \ldots \ldots(8)
$$

Finally a scaling property which is important, especially for active transducers like piezoelectric accelerometers, is the amount of energy available for transduction when a certain vibration is present. For a certain instantaneous velocity $v$ of the accelerometer the kinetic energy stored in the motion of the seismic mass $M$ is

$$
E_{\text {Kinetic }}=\frac{1}{2} M v^{2}
$$

These scaling properties makes it necessary to look at effects which were not of any concern hitherto.

\section{$<2.1>$ Thermal noise}

Mechanical motion The thermal motion (displacement) of the seismic mass can be described as follows (see e.g. $\left.{ }^{(2)}\right)$ :

$$
\begin{gathered}
\sigma_{d T}{ }^{2}=4 K T \Delta f \frac{1}{M \omega_{0}{ }^{2} Q}\left[1-\left(\frac{\omega}{\omega_{0}}\right)^{2}\right]^{2}+\left(\frac{\omega}{Q \omega_{0}}\right)^{2} \cdots(10) \\
\begin{array}{c}
\omega \ll \omega_{0} \\
\sigma_{d T}{ }^{2}=4 K T \Delta f \frac{1}{M \omega_{0}{ }^{2} Q}
\end{array}
\end{gathered}
$$

or if using

$$
F=k x=\omega_{0}^{2} M
$$

it can be expressed as force:

$$
\sigma_{F T}^{2}=4 K T \Delta f \frac{\omega_{0}{ }^{2} M}{Q}
$$

Where $K$ is Boltzmanns constant, $\Delta f$ the frequency band of observation centered around $\omega, T$ the absolute temperature and $Q$ the quality factor of the resonance.

This gives the following proportionalities:

$$
\sigma_{d T} \propto \frac{1}{\sqrt{M}} \propto \frac{1}{\sqrt{L^{3}}}
$$

and

$$
\sigma_{F T} \propto \sqrt{M} \propto \sqrt{L^{3}} \ldots \ldots
$$

and in both cases the signal to noise ratio becomes:

$$
\frac{S}{N} \propto \sqrt{L^{3}}
$$

which means that by scaling the transducer size as proposed it becomes increasingly difficult to get a proper signal to thermal noise ratio. Till today the electronic noise has been by far the dominant factor, but going to the micromechanical magnitudes, we will reach the thermal limit provided the electronic noise does not increase, which is normally not the case.

\section{Resistance noise}

The well known Johnson noise or thermal noise of a resistor

$$
u_{n}=\sqrt{4 K T R \Delta f}
$$

should naturally not be forgotten.

$<2.2>$ Quantum effects Having dealt with the ther- 
mal noise, we also have to evaluate contributions from quantum effects.

An ideal capacitor in the form of a piezoelectric material or a variable capacitor is limited by the smallest available charge, the electron charge:

$$
\mathrm{e}=1.6 \cdot 10^{-19} \mathrm{C}
$$

This might be a limiting factor for piezoelectric transducers which are basically capacitors (assuming no losses).

Linked to a current is the so called shot noise which is valid for cases where the electrons can be considered free

$$
J_{n}=\sqrt{2 e I \Delta f} \ldots \ldots . . . . .
$$

For the tunnelling effect mentioned later this is the most important electrical noise source.

For resistors which are not pure metallic, different more complicated mechanisms produce current noise. An expression including most phenomena will have the following form (adapted from ${ }^{(3)}$ )

$$
v^{2}=\Delta f V_{0}^{2}\left(A \frac{1}{f}+B \frac{1}{1+(2 \pi \tau)^{2} f^{2}}\right) \cdots . .
$$

where $v$ is the noise voltage over the resistance, $V_{0}$ the $\mathrm{DC}$ voltage across the resistor, $\tau$ a relaxation time constant and $A$ and $B$ constants depending on dimensions and materials. An interesting observation for scaling of transducers with the resistance kept constant is that

$$
A \propto A_{c}^{-2} \propto L^{-4} \quad \text { and } \quad B \propto l^{-2} \propto L \ldots
$$

$A_{c}$ being the cross-section of the resistor and $l$ the length.

This shows that especially the $1 /$ f noise can become a serious problem when downscaling is made.

In some cases a term following $1 / \sqrt{ } f$ will also be present, but this has no clear dimensional dependence.

The electronics used for feedback/readout may or may not be able to reach the limits given by the intrinsic noise stated here.

\section{Practical implications}

To put these "new" parameters into perspective in conjunction with possible applications of today, we will evaluate the effects if we want to make a dedicated modal analysis accelerometer for light structures.

A set of desirable characteristics could be:

Dynamic range ( $1 \mathrm{~Hz}$ bandwidth) $100 \mathrm{~dB}$

Frequency range $( \pm 10 \%)$

$\left(1 \mathrm{~mm} / \mathrm{s}^{2}\right.$ to $\left.100 \mathrm{~m} / \mathrm{s}^{2}\right)$

Nonlinearity

$0.1 \mathrm{~Hz}$ to $2 \mathrm{kHz}$

Max. cross-axis sensitivity

Max. $1 \%$ at $100 \mathrm{~ms}^{2}$

Weight

$<5 \%$

Maximum shock

$<1$ gram

$>20000 \mathrm{~m} / \mathrm{s}^{2}$

\section{$<3.1>$ Resonance frequency and $Q$ factor The res-}

onance frequency should be around $5 \mathrm{kHz}$, which is reasonably above the frequency range of interest. To keep the phase of the measured signal in the whole frequency range of interest lower than approx. $1^{\circ}$, the $Q$-factor should be $>20$, which keeps the phase shift "concentrated" around the resonance frequency but does however prevent long duration ringing when the resonance is excited.

$<3.2>$ Noise level specifications A few words may be said about the specified noise level.

The requirement for the modal analysis accelerometer considered is that the noise level for all frequencies between $1 \mathrm{~Hz}$ and $1 \mathrm{kHz}$ is $10^{-3} \mathrm{~m} / \mathrm{s}^{2} / \sqrt{\mathrm{Hz}}$ or lower. An accelerometer with this spectral noise density and a white-noise spectrum is considered useful. However, one problem is that preamplifiers show a frequency dependent noise which has to be dealt with.

It should be noted that this criterion is only relevant for electrical noise, and not for the mechanical-thermal noise level nor for the shot noise, which both are white in the working range as shown earlier (Eq. 10.).

\section{Examples}

Six different transduction principles, that are considered as the most relevant for silicon-based accelerometers, will be analysed.

First, the accelerometers based on strain sensing are discussed. These are the piezoresistive, the piezoelectric, the resonant and the optical strain gauge accelerometer.

Next, the displacement-sensing accelerometers are discussed: capacitive accelerometers (with and without forced balancing) and accelerometers based on electron tunnelling.

Finally a comparison of the different transduction principles is made.

$<4.1>$ Strain-sensing accelerometers Strain-sensing accelerometers have proven to be successful over many years. Bruiel \& Kjaer's piezoelectric accelerometers are based on strain sensing (or stress sensing). An advantage of strain sensing is that the accelerometer structures can actually be very stiff, thereby obtaining a robust structure with a high resonance frequency. These properties are typical for Brül \& Kjaer's accelerometers, where it is demonstrated that the stiff structure still allows measurement of low-level vibrations in a high dynamic range. The first silicon micromachined accelerometer, the famous device of Roylance and Angell ${ }^{(1)}$, which was developed at Stanford University in 1979, was based on another strain-detection principle, piezoresistive detection.

Two relatively new strain detection principles are the resonant principle, where the resonance frequency of a small bridge structure is used as a measure for the strain, and the optical strain gauge based on Bragg grating, giving an optical frequency shift when strained ${ }^{(4)}$. 
The detection principles will be discussed in the next sections.

\section{Piezoresistive detection}

The piezoresistive effect is widely used for silicon sensors. The effect is known to be linear up to stress levels which are close to the yield strength of silicon, whereas in the lower end the resolution of the strain gauge is determined by the Johnson noise in the piezoresistors ${ }^{(1)}$. The linearity of the piezoresistive effect can give a high dynamic range of the accelerometer.

\section{Dynamic range of the Wheatstone bridge}

The maximum measurable acceleration is usually determined by other nonlinearities than caused by the piezoresistive effect itself. The piezoresistors are used in a Wheatstone bridge configuration. The output voltage $\Delta U$ of a Wheatstone bridge with one active resistor is

$$
\Delta U=\frac{\Delta R}{(4 R+2 \Delta R)} U_{0} \ldots \ldots \ldots
$$

where $U_{0}$ is the $\mathrm{DC}$ supply voltage, $R$ is the resistor value and $\Delta R$ is the resistance change. It follows that the Wheatstone bridge is only linear as long as $\Delta R<<2 R$. This criterion can be written as a criterion for the maximum strain $\mathrm{e}$ that can be measured using the definition of the gauge factor G:

$$
\frac{\Delta R}{R}=G \varepsilon
$$

If we take a nonlinearity of $1 \%$ on $\Delta U$ as the upper limit for detection, then (from 22.) $\Delta R / R<<0.02$. The gauge factor is dependent on the doping level of the silicon, and can be up to 200 for monocrystalline silicon. For polycrystalline silicon a typical gauge factor is around $20^{(5)}$. This gives a maximum measurable strain of $10^{-3}$ for polycrystalline silicon strain gauges, and $10^{-4}$ for monocrystalline silicon.

The resolution of the strain gauge is limited by the thermal noise of the piezoresistors, which typically have values between 1 and $10 \mathrm{kOhm}$. A typical value for the thermal noise of a piezoresistor is then (eq. 17.) approximately $10 \mathrm{nV} / \sqrt{\mathrm{Hz}}$. If the DC supply voltage of the Wheatstone bridge is $5 \mathrm{~V}$, and assuming one active piezoresistor, then the minimum relative resistance change that can be detected is $8 \cdot 10^{-9}$. Using the same gauge factors for polycrystalline and monocrystalline silicon as mentioned above, the minimum strains that can be resolved are $4 \cdot 10^{-10}$ and $4 \cdot 10^{-11}$, respectively.

From this it follows that the dynamic range of a Wheatstone bridge with one active piezoresistor is limited to 128 $\mathrm{dB}$, because of the thermal resistor noise and the requirement of a maximum nonlinearity of $1 \%$. Note that the Wheatstone bridge gets twice as sensitive by using two active resistors, and four times as sensitive by using four active resistors (it is assumed that four resistors of the same magnitude is used in all cases!). This results in noise levels which is 6 and
$12 \mathrm{~dB}$ lower, respectively. For the bridge with two active resistors the nonlinearity criterion is the same as for the bridge with one active resistor. The bridge with four active resistors does not show the nonlinearity of the Wheatstone bridge, as mentioned above. In practice, nonlinearities will for example be introduced by the nonideal placement (alignment errors) of the piezoresistors on the bending elements, causing the resistance changes to be slightly different for each strain sensing element.

\section{The suspension beams: Nonlinearity and cross-axis sensitivity}

Another type of nonlinearity appears when the piezoresistive strain gauges are used in a an accelerometer construction. Usually the strain gauges are used in a beam bender construction, since the highest sensitivity is obtained in this way. (examples: Christel et al. ${ }^{(6)}$, Roylance and Angell ${ }^{(1)}$, Crazzolara et al. ${ }^{(7)}$, Terry et al. $\left.{ }^{(8)}\right)$. However, such a beam bender construction can put an upper limit to the detection, by introducing a nonlinearity caused by stretching of the beam material at large beam deflections. This is the case when the seismic mass is suspended in a stiff frame by beams at opposite sides. An example of such a construction is shown in Fig. 1 (from Allen et al. ${ }^{\{2\}}$ ).

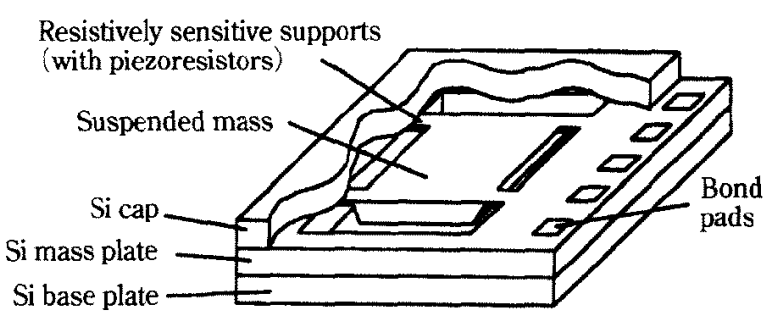

Fig. 1. Example of piezoresistive accelerometer with four beam suspension. From Allen et al. ${ }^{(12)}$.

A beam bender construction based on a single cantilever beam is not sensitive to this type nonlinearity. This nonlinearity has been demonstrated by Christel et al. from Lucas Novasensor ${ }^{(6)}$, where cantilever- and fourbeam accelerometers, both with a $2 \mathrm{~g}$ range, were compared and showed errors of $1 \%$ and $25 \%$ respectively at $8 \mathrm{~g}$ acceleration,. A disadvantage of the cantilever construction as used by Christel et al. is that it cannot distinguish between in-plane and out-of-plane accelerations, because the center of gravity of the seismic mass is not positioned in the plane of the cantilever beam. Such cantilever beam constructions can be fabricated, by for example adding an electroplated gold mass on the silicon seismic mass (Heuberger ${ }^{(9)}$ ), but this usually complicates the fabrication process. Another way to get around the problem of the nonlinearity of beam bender constructions is to use the piezoresistors in a push-pull configuration, as for example demonstrated by Suminto ${ }^{(1)}$. A disadvantage of using strain gauges in a push-pull configuration is the lower sensitivity which limits the applications to cases where the acceleration 
levels are relatively high.

\section{The measurement amplifier}

Due to the low output impedance of a piezoresistive accelerometer, the component can be connected directly to a measurement amplifier. The typical noise level of a measurement amplifier, is around $10 \mathrm{nV} / \sqrt{\mathrm{Hz}}$. However, to obtain this low noise level, the gain of the first amplifier stage should be $40 \mathrm{~dB}$ and DC coupled. This implies that the DC component in the accelerometer output signal should be less than $50 \mathrm{mV}$, in order not to drive the DC output of the first amplifier stage to the rail. Consequently, the sensitivity of the accelerometer should be less than $50 \mathrm{mV} / \mathrm{g}$, to avoid that the earth's gravitational field causes this output signal.

Other causes for a DC component in the accelerometer's output signal are unbalance of the Wheatstone bridge, and nonlinearities in the sensitivity of the device (Christel et al. $)^{(6)}$. However, the response to these effects can be kept under $50 \mathrm{mV}$. Of course the DC output can be decoupled with a series capacitor between the accelerometer and the amplifier, but then the noise level is suddenly determined by the 1 MOhm typical input resistor of the measurement amplifier, causing the noise level to increase with $20-30 \mathrm{~dB}$.

\section{Performance of accelerometers from the literature}

Since the expected noise level of the piezoresistors and of the preamplifier have to be added, giving $14 \mathrm{nV} / \sqrt{\mathrm{Hz}}$ ideally, a total noise level of $20 \mathrm{nV} / \sqrt{\mathrm{Hz}}$ is taken as a fixed value for analysing the performance of accelerometers from the literature. The resolution of the accelerometer is then this noise level, divided by its sensitivity.

To evaluate the performance of an accelerometer in an objective way, not only noise level and dynamic range should be compared, but the resonance frequency should be taken into account as well. In table 1 data from several published accelerometer designs are listed.

Assuming that one can resolve $20 \mathrm{nV} / \sqrt{\mathrm{H} z}$, the dynamic ranges of the listed accelerometers are high. For modal analysis purposes, the accelerometers of Crazzolara and Tschan do have a suitable resonance frequency and sufficient resolution. The accelerometer of Roylance and Angell does have sufficient resolution, but the construction cannot be made stiffer to increase the resonance frequency. With the accelerometer from Barth et al. the sensitivity can be lowered a factor of 100 , thus obtaining a resonance frequency of $5 \mathrm{kHz}$ and a resolution of $0.2 \mathrm{~mm} / \mathrm{s}^{2}$. The response to the earth's gravitational field is less than $50 \mathrm{mV}$ for accelerometers with a proper resonance frequency.

\section{Temperature sensitivity}

A typical problem with piezoresistive sensors is the temperature sensitivity. Both the offset and the sensitivity do change with temperature. Typically the temperature coeffcients of offset (TCO) and sensitivity (TCS) are $0.2-0.3 \% / \mathrm{C}$, when no measures are taken to compensate for this. With temperature compensation used, these values can be brought down to $2 \%$ over a temperature range from -40 to $+85 \mathrm{C}^{(14)}$. However, this requires accurate trimming of each individual component adding to the cost.

\section{Summary}

Summarising, piezoresistive accelerometers can have a high dynamic range, in combination with a sufficiently high resonance frequency for modal analysis applications. The resolution that can be obtained in practice is expected to be sufficient, when using a low noise measurement amplifier, that can handle the DC response of the accelerometer. A drawback of piezoresistive detection is the temperature sensitivity, although effective temperature compensation schemes have been developed.

\section{Piezoelectric detection}

The only micromechanical piezoelectric accelerometer reported in the literature is the device from Chen et al ${ }^{(15)} \mathrm{de}$ veloped at Berkeley/Rockwell in 1982, and shown in Fig. 2. It was an open-loop, cantilever-type accelerometer, based on sputtered $\mathrm{ZnO}$, and with an on-chip MOS preamplifier. In 1994, a theoretical study was done at B\&K to investigate the possibility of a triaxial $\mathrm{ZnO}$-based accelerometer ${ }^{(16)}$.

The next section is divided into three parts. First, the noise

\begin{tabular}{|l|c|c|c|c|c|c|}
\hline Authors & $\begin{array}{c}\text { Working } \\
\text { range } \\
\left(\mathrm{m} / \mathrm{s}^{2}\right)\end{array}$ & $\begin{array}{c}\text { Sensitivity } \\
(5 \mathrm{~V} \text { suppiy }) \\
\left(\mu \mathrm{V} / \mathrm{ms}^{-2}\right)\end{array}$ & $\begin{array}{c}\text { Resolution } \\
(\mathrm{m} / \mathrm{s} 2 / \sqrt{\mathrm{Hz}})^{\star}\end{array}$ & $\begin{array}{c}\text { Dynamic } \\
\text { range } \\
(\mathrm{dB})\end{array}$ & $\begin{array}{c}\text { Resonance } \\
(\mathrm{kHz})\end{array}$ & $\begin{array}{c}\text { Type } \\
\text { Roylance and Angell }^{(1)}\end{array}$ \\
\hline Barth et al. $^{(1)}$ & 1960 & 25 & $8 \cdot 10^{-4}$ & 128 & 2.3 & $\mathrm{C}$ \\
\hline Crazzolara $^{(7)}$ & 19.6 & 10000 & $2 \cdot 10^{-6}$ & 140 & 0.5 & $\mathrm{C}$ \\
\hline Allen et al. $^{(12)}$ & 3330 & 20 & $1 \cdot 10^{-3}$ & 130 & 7 & $\mathrm{D}$ \\
\hline Tschan $^{(13)}$ & 196 & 255 & $8 \cdot 10^{-5}$ & 128 & 2 & $\mathrm{Q}$ \\
\hline
\end{tabular}

Table 1. Performance of accelerometers reported in the literature

* a noise level of $20 \mathrm{nV} / \sqrt{\mathrm{Hz}}$ has been assumed

Type: $\mathrm{C}=$ cantilever, $\mathrm{D}=$ two beam bridge type, $\mathrm{Q}=$ four beam bridge type 
equivalent stress of a piezoresistive and a piezoelectric strain gauge are compared. Next, previous calculations on $\mathrm{ZnO}$ accelerometers are critically reviewed, and finally PZT benders are investigated further.

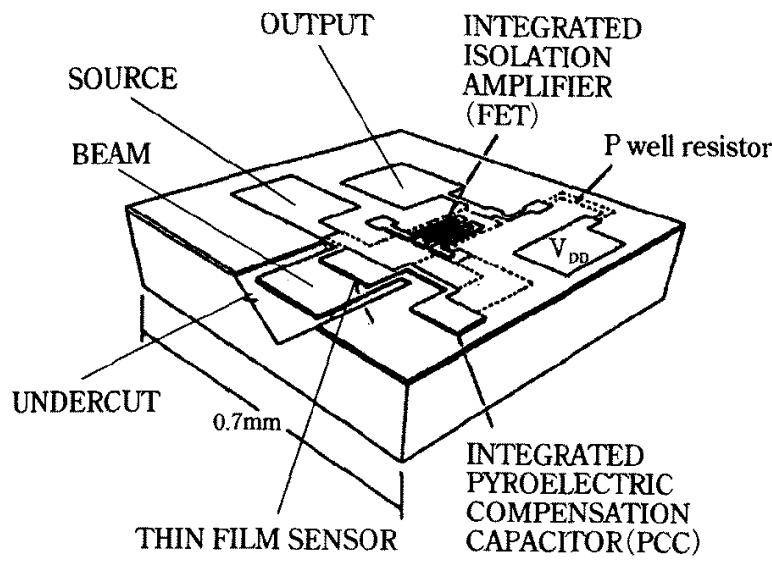

Fig 2. Piezoelectric $\mathrm{ZnO}$ based accelerometer, after ${ }^{(15)}$.

\section{Piezoresistive vs. piezoelectric strain gauges}

A comparison, valid for some applications, between the piezoresistive and the piezoelectric transduction principle can be made by comparing the signal-to-noise ratio of two strain gauges. A rectangular strain gauge is assumed, which is loaded by a uniform, uniaxial stress $\sigma_{x}$.

\section{Piezoelectric}

For the piezoelectric strain gauge it can easily be shown that the generated charge $Q$ in the $z$-direction, per stress-unit (i.e. $\mathrm{Pa}$ ), is

$$
\frac{Q}{\sigma_{x}}=\frac{d_{3 t} t C_{x}}{\varepsilon_{33}}
$$

where $t$ is the thickness of the piezoelectric material, $C_{z}$ is the capacitance of the piezoelectric layer, and $\varepsilon_{33}$ is the permittivity of the piezoelectric film in the $z$-direction $\left(\varepsilon_{33}=\varepsilon_{0}\right.$. $\left.\varepsilon_{n}\right)$.

In principle, if the leakage current through a piezoelectric transducer is low, all the noise is generated by the charge amplifier. The amplifier noise is usually modelled as a whitenoise voltage source $e_{n}$ at the amplifier input node. The equivalent charge noise $q_{n}$ at the amplifier input can then be calculated. The result is

$$
q_{n}=e_{n}\left(C_{f}+C_{z}\right) \ldots \ldots \ldots-(25)
$$

where $C_{f}$ is the feedback capacitance in the charge amplifier, which determines the gain. At a high-gain setting, the feedback capacitance is usually much smaller than $C_{x}$. Note that parasitic capacitances have been neglected.

Assuming no parasitic capacitances, and a high-gain setting of the charge amplifier, the noise equivalent stress in $\mathrm{Pa}$ becomes

$$
N=\frac{\sigma_{x} q_{n}}{Q}=\frac{\varepsilon_{33} e_{n}}{d_{31} t}
$$

Note that the important parameter for the piezoelectric material is the ratio $d_{31} / \varepsilon_{33}$ This ratio is approximately $-0.060 \sim \mathrm{m}^{2} / \mathrm{C}$ for $\mathrm{ZnO}$, and $-0.015 \sim \mathrm{m}^{2} / \mathrm{C}$ for PZT2 (data from Berlincourt ${ }^{(9)}$.

If a B\&K 2626 charge amplifier is used, the noise source $e_{n}$ is approximately $1.2 \times 10^{8} \mathrm{~V} / \sqrt{\mathrm{Hz}}$. Assuming a $1 \mu \mathrm{m}$ thick piezoelectric layer, the noise becomes $0.2 \mathrm{~Pa} / \sqrt{ } \mathrm{Hz}$ for $\mathrm{ZnO}$, and $0.8 \mathrm{~Pa} / \mathrm{JHz}$ for $\mathrm{PZT} 2$.

\section{Piezoresistive}

The output signal of the piezoresistive strain gauge can easily be found with the equations given in the previous section. Assuming a Wheatstone bridge with 4 active element (highest sensitivity), the output signal $u$ per unit stress becomes

$$
\frac{u}{\sigma_{x}}=\frac{G U_{0}}{E} \ldots \ldots . . . . . .
$$

where $G$ is the gauge factor, $U_{0}$ is the supply voltage and $E$ is the Young's modulus of the strain gauge material $\left(1.7 \times 10^{11}\right.$ $\mathrm{N} / \mathrm{m}^{2}$ for silicon in the $\langle 110\rangle$-direction).

The noise level is given by the well-known Johnson noise equation stated in the previous section. The noise equivalent stress in $\mathrm{Pa} / \sqrt{\mathrm{Hz}}$, at the Wheatstone bridge output becomes

$$
N=\frac{u_{n} \sigma_{x}}{u}=\frac{E \sqrt{4 K T R}}{G U_{0}}
$$

where $K$ is Boltzmann's constant $\left(1.38 \times 10^{-23} \mathrm{~J} / \mathrm{K}\right), T$ is the absolute temperature, and $R$ is the bridge resistance.

At a supply voltage of $5 \mathrm{~V}$, a temperature of $293 \mathrm{~K}$, and assuming a gauge factor of 200 and a resistance of $1 \mathrm{k} \Omega$, the noise equivalent stress becomes $0.67 \mathrm{~Pa} / \sqrt{\mathrm{Hz}}$.

\section{Conclusion}

Comparing the noise equivalent stress of the piezoelectric and the piezoresistive strain gauges, it can be concluded that a PZT-based piezoelectric strain gauge is as good as a piezoresistive strain gauge (other types of PZT with a higher $d_{31} / \varepsilon_{33}$ ratio do exist, e.g. $-0.021 \mathrm{~m}^{2} / \mathrm{C}$ for PZT-5H $\left.{ }^{(17)}\right)$.

It appeared that zinc oxide-based strain gauges should be able to give lower noise levels.

In a theoretical study, calculations of sensitivity and nonlinearity were done on a $\mathrm{ZnO}$-based triaxial accelerometer ${ }^{(16)}$. Noise level simulations were done on an ASIC charge amplifier, that should be built together with the accelerometer in a package. In the study, it was concluded that the noise level requirements could be fulfilled.

However, this conclusion was based on a noise value at $100 \mathrm{~Hz}$. Calculated noise spectra show that the noise below $1000 \mathrm{~Hz}$ shows a $1 / f$ dependence, implicating that at $1 \mathrm{~Hz}$ the dynamic range is $40 \mathrm{~dB}$ lower. Thus, the accelerometer can possibly fulfil broadband-noise requirements, but for modal analysis it is more desirable that the noise-level is low for all frequencies.

Reducing the noise by $40 \mathrm{~dB}$ is difficult. Increasing the seis- 
mic mass and lowering the capacitance did not prove it possible to gain more then maybe $20 \mathrm{~dB}$ at the most.

It can be concluded that measuring vibrations below $100 \mathrm{~Hz}$ is not possible down to the required detection level with a $\mathrm{ZnO}$-based accelerometer as described in ${ }^{(16)}$. The reported designs can be optimised, but not enough to achieve a $40 \mathrm{~dB}$ improvement of the signal-to-noise-ratio. A comparison between PZT- and $\mathrm{ZnO}$-strain gauges shows that a PZT-based accelerometer will show a considerably higher sensitivity, but not a better resolution.

\section{Further comments}

These considerations have all been based on the assumption that the piezoelectric material is used as a strain gauge in the same way as a piezoresistive element. This is however somewhat misleading, because the piezoresistive element is supplied with a current to measure the deformation, and is then best used when placed at maximum strain points on a given structure. The structure can then be designed for resonance frequency etc.

When using piezoelectric transduction it must however be remembered that it is an active transduction of vibration energy into electrical energy, and therefore as much as possible of the energy must be "guided" to the piezoelectric material. In this case it means that the beam shall be made from the piezoelectric material to as large an extend as possible.

To evaluate the possibilities we can use the formulas given for macroscopic bimorphs and see what the scaling could bring.

C.P. Germano ${ }^{(18)}$ gives the formulas

$$
\begin{aligned}
& S_{,}=\frac{3}{2} \frac{g_{31} l}{w t} \ldots \ldots \ldots \\
& k=\frac{Y_{11}{ }^{D} w t^{3}}{4 l^{3}} \ldots \ldots . . . . . .
\end{aligned}
$$

and

$$
C_{e}=\frac{\varepsilon_{33} h w}{t}
$$

for the voltage sensitivity, spring stiffness and capacitance of a series connected rectangular bimorph. $l, w$ and $t$ are the length, width and thickness of the bender in the one end clamped condition. $g_{31}=d_{31} / \varepsilon_{33} . Y_{11}^{D}$ is the elastic constant $\left(\mathrm{N} / \mathrm{m}^{2}\right)$. If we use the dimensions $l, w, t=1 \mathrm{~mm}, 0.2 \mathrm{~mm}$, $0.2 \mathrm{~mm}$ and $10 \cdot 10^{-3} \mathrm{Vm} / \mathrm{N}$ for $g_{31}, 6.9 \cdot 10^{10} \mathrm{~N} / \mathrm{m}^{2}$ for $Y_{11}^{D}$ and $2000 \cdot 8.85 \mathrm{pF} / \mathrm{m}$ for $\varepsilon_{33}$ we get $S_{v}=375 \mathrm{~V} / \mathrm{N}$ which with a seismic mass of $3 \mathrm{mg}$ gives a vibration sensitivity of $11 \mathrm{mV} / \mathrm{g}$. The capacitance becomes $C_{\varepsilon}=18 \mathrm{pF}$ and the resonance frequency $15.3 \mathrm{kHz}$.

Using a maximum strain of $20 \cdot 10^{6} \mathrm{~N} / \mathrm{m}^{2}$ the maximum vibration level before fracture can be calculated to be approximately $900 \mathrm{~g}$ using the formula for strain from earlier.

This leads to a noise level of approximately $1 \mu \mathrm{g}$, and therefore nearly $180 \mathrm{~dB}$ useful dynamic range for the transducer alone (nonlinearity will reduce this number $10-20 \mathrm{~dB}$ if a $2 \%$ limit is used). If the swing is limited to $5 \mathrm{~V}$ and $18 \mathrm{pF}$ is used as feedback, then the useful dynamic range at the output of a charge amplifier is $168 \mathrm{~dB}$, and the noise level $0.02 \mathrm{~mm} / \mathrm{s}^{2}$. This is sufficiently low to fulfil the modal accelerometer requirements stated in the beginning.

To fulfil the $1 \mathrm{~Hz}, 10 \%$ lower limiting frequency requirement a $30 \mathrm{G} \Omega$ resistor is needed in parallel to the $18 \mathrm{pF}$ feedback capacitor. This could be difficult to realise. A suitable trade-off between $1 / \mathrm{f}-$ noise, lower limiting frequency and feedback resistor must be made.

The noise level of $1 \mu \mathrm{g}$ approaches also the quantum noise level stated earlier, because the stated sensitivity corresponds to a charge sensitivity of $0.2 \mathrm{pC} / \mathrm{g}$ and this gives a charge of $2 \cdot 10^{19} \mathrm{C}$ at $1 \mu \mathrm{g}$ which is close to the electron charge of $1.6 \cdot 10^{19} \mathrm{C}$.

\section{Conclusion}

The conclusion to be drawn from this section is that PZT benders in miniature format should be a viable choice for a modal accelerometer.

This part of micromachining is however not studied in sufficient detail to guarantee that the technology can be scaled without problems. Most of the work done on PZT materials is aimed at thin films which are much thinner than the dimensions indicated here (typically $<1 \mu \mathrm{m}$ ).

\section{Resonant strain detection}

The third way to detect strain is the resonant strain gauge.

The strain is indicated by a change in the resonance frequency of a vibrating beam or similar structure. The resonant frequency is typically in the range 50 to $500 \mathrm{kHz}$.

The modulation can be up to $25 \%$ without introducing serious distortion although the effect is quadratic.

Quality factors (QS) in the range from 200 in air to 100,000 in vacuum has been used. $A$ high $Q$ is naturally desirable if high resolution is needed. This imposes special requirements to the packaging which might be difficult to obtain for extended timespans.

The resonant gauge has the potential of increasing the resolution because of the basic FM modulation property giving a signal to noise improvement proportional to the ratio of modulation to detection bandwidth i.e.

$$
\frac{S}{N} \ldots \alpha \ldots \frac{\Delta f_{\text {Modulation }}}{\Delta f_{\text {Detection }}} \cdots \ldots . . . .
$$

If a $2 \mathrm{kHz}$ bandwidth is needed and $20 \mathrm{kHz}$ (i.e. $\pm 10 \mathrm{kHz}$ ) modulation can be obtained a $20 \mathrm{~dB}$ higher $\mathrm{S} / \mathrm{N}$ ratio can be obtained.

Most reports are giving $\mathrm{DC}$ measurements, maybe corre sponding to a bandwidth of $0.1 \mathrm{~Hz}$ and therefore a very high resolution and large dynamic range.

The only vibration test reported to our knowledge were made at $200 \mathrm{~Hz}$ and gave a $60 \mathrm{~dB}$ dynamic range and $50 \mathrm{mg}$ resolution ${ }^{19 i}$. The principal part of the construction is shown 
in Fig. 3.

The FM demodulators used today will hardly give more than 80 to $100 \mathrm{~dB}$ dynamic range for the desired $2 \mathrm{kHz}$ bandwidth.

To our knowledge, the only commercially available resonant accelerometer is made by the Norwegian company Sen

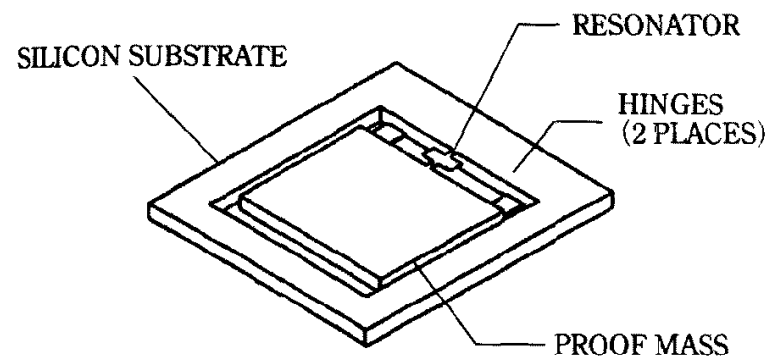

Fig. 3. Hinged mass with resonant beam construction, after Roszhart et al. ${ }^{(19)}$

soNor ${ }^{(20,21)}$. Their SA30 accelerometer is a component aiming at the airbag sensor market. With this component, the resonant detection principle is primarily used to provide a continuous self test during operation of the device, thereby considerably improving the reliability of the accelerometer.

Results of development of high-performance resonant accelerometers were presented by Roszhart et al. at Kearfott/IC Sensors ${ }^{(19)}$ and Honeywell ${ }^{(22)}$. These accelerometers are developed for navigation purposes, where a high resolution is required, whereas the required frequency range is low.

\section{Optical detection}

Several different schemes have been devised to use light to detect displacements, most of which falls short of fulfilling our wish to resolve $10^{-12} \mathrm{~m}$ or $10^{-6}$ of a wavelength related to detection of acceleration.

However, a promising method based on a Bragg grating embedded in an optical waveguide acting as a strain gauge, has recently been described (4).

The principle of operation is based upon the Bragg condition for the dominant wavelength component $\lambda_{b}$ of the light reflected by the grating, It is given by the formula

$$
\lambda_{b}=2 \Lambda n_{\text {eff }}
$$

where $\Lambda$ is the grating period and $n_{\mathrm{eft}}$ is the effective index of refraction.

The strain sensitivity is given by

$$
\frac{\Delta \lambda_{b}}{\lambda_{b}}=\left(1-p_{e}\right) \varepsilon \ldots . . . .
$$

Where $p_{e}$ is the photoelastic strain coefficient ( 0.22 for pure silica). The expected sensitivity for an accelerometer with a $13 \mathrm{kHz}$ resonance frequency is $1.7 \cdot 10^{-14} \mathrm{~nm} / \mathrm{ms}^{-2}$, and the resolution which can be obtained is $4.4 \cdot 10^{-3} \mathrm{~ms}^{-2}$. Reducing the resonance frequency to half the value will give the desired resolution of $10^{-3} \mathrm{~ms}^{2}$.

The principle including the detection method is quite new (1992) and many technological problems have to be solved before a product can reach the market.

\section{$<$ 4.2> Capacitive detection with force-balancing} With the capacitive detection principle, the displacement of the seismic mass is measured, instead of the strain in a suspension beam. Advantages of capacitive detection are the high sensitivity and the low temperature sensitivity. Since the capacitance between the seismic mass and a second fixed plate is inversely proportional to the distance, a capacitive accelerometer is non-linear. Capacitive accelerometers are often used in combination with a force-balancing system for linearization. Another advantage of the feedback system is the extended frequency range. Without the use of a feedback system, the strong damping due to the narrow airgap causes the bandwidth to be much less than the resonance frequency of the seismic mass. An example of a capacitive accelerometer construction is shown in figure 4 .

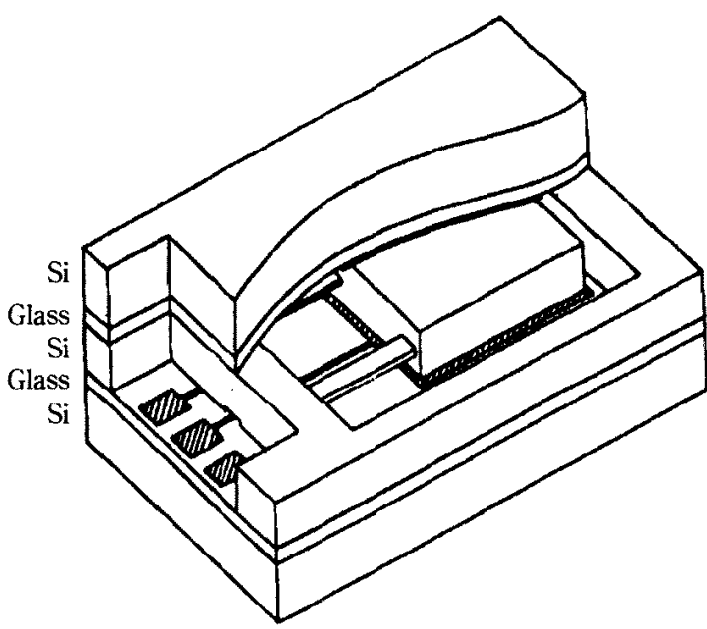

Fig. 4. Capacitive accelerometer, after Rudolf et al. ${ }^{(24)}$.

\section{Working range}

One upper limit of the dynamic range is set by the feedback system. The maximum acceleration is given by the force acting on the seismic mass (mass $\times$ acceleration) that still can be compensated by electrostatic forces, which depend on the maximum voltage $\Delta V$ that the feedback amplifier can generate, and on geometrical factors (the electrode area $A$ and the plate separation $d$ ). The following expression for the maximum acceleration is given by Kiihnel and Sherman ${ }^{(23)}$ :

$$
a=\frac{2 \varepsilon_{0} A}{M d^{2}} V_{p} \Delta V \ldots \ldots \ldots
$$

where $V_{p}$ is the $\mathrm{DC}$ polarisation voltage between the capacitor plates, and $\varepsilon_{0}$ is the vacuum permittivity.

In Table 2 data are given for the $\mathrm{ADX} \perp 50$ accelerometer from Analog Devices and the A-SMAC accelerometer from 
CSEM. ${ }^{(24)}$. These accelerometers have measured working ranges of $80 \mathrm{~g}$ and $0.1 \mathrm{~g}$, respectively. The calculated limits set by the feedback system for these accelerometers are 250 $\mathrm{g}$ and $0.2 \mathrm{~g}$. The large difference between the calculated and the measured working range of the ADXI50 is due to the amplifier output voltage, which is limited to the output voltage generated at $80 \mathrm{~g}$ acceleration.

\begin{tabular}{|l|c|c|}
\hline Transducer type. & A-SMAC & ADXL50 \\
\hline Seismic mass $(\mathrm{mg})$ & 14.7 & $0.16 \cdot 10^{-3}$ \\
\hline Air gap $(\mu \mathrm{m})$ & 7 & 1.3 \\
\hline Capacitance $(\mathrm{pF})$ & 20 & 0.1 \\
\hline Polarisation voltage $(\mathrm{V})$ & 2.5 & 1.6 \\
\hline Voltage swing $(\mathrm{V})$ & 2.0 & 1.6 \\
\hline Measured data: & & \\
\hline Sensitivity $(\mathrm{V} / \mathrm{g})$ & 5 & 0.020 \\
\hline Working range $(\mathrm{g})$ & 0.1 & 80 \\
\hline Noise level $(\mathrm{g} / \mathrm{VHz})$ & $0.1-0.5 \cdot 10^{-5}$ & $4.9 \cdot 10^{-3}$ \\
\hline Resonance freq. $(\mathrm{kHz})$ & 0.126 & 20 \\
\hline Q factor & not specified & 5 \\
\hline Calculated data: & & \\
\hline Sensitivity $(\mathrm{V} / \mathrm{g})$ & 4.5 & 0.0001 \\
\hline Working range $(\mathrm{g})$ & 0.2 & 250 \\
\hline Thermal noise $(\mathrm{g} / \mathrm{VHz})$ & $0.095 \cdot 10^{-6}$ & $0.16 \cdot 10^{-3}$ \\
\hline
\end{tabular}

Table 2. Data for two capacitive accelerometers.

\section{Noise level}

The resolution of the accelerometer is given by the preamplifier noise divided by the sensitivity. To calculate the resolution of the accelerometer, the preamplifier noise has to be calculated. A full discussion of preamplifier noise is beyond the scope of this paper. However, a general rule for preamplifiers for this type of capacitive sensors is that the preamplifier noise decreases if the input capacitance increases, where the input capacitance is the sum of the accelerometer capacitance and the parasitic capacitances. It should be noted that when one keeps the input capacitance constant, and changes the ratio between the accelerometer capacitance and the parasitic capacitance, the noise will remain constant, but the signal-to-noise ratio gets worse if the parasitic capacitance becomes larger. The CSEM preamplifier shows a noise level of

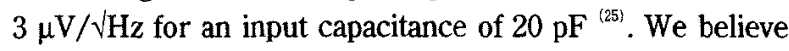
that this noise level can be considered as representative for the current state-of-the-art, and we will use this value for further analysis.

Kühnel and Sherrnan ${ }^{(23)}$ derive that the sensitivity $S$ of a capacitive accelerometer, that is used in a differential readout configuration, is:

$$
S=\frac{V_{p}}{\omega_{0}^{2} d}
$$

where $\omega_{9}$ is the vacuum resonance frequency. As can be seen in Table 2 , this equation predicts a sensitivity of 100 $\mu \mathrm{V} / \mathrm{g}$ for the ADXI.50 accelerometer, and a sensitivity of 4.5 $\mathrm{V} / \mathrm{g}$ for the A-SMAC. The later value agrees well with the measured value of $5 \mathrm{~V} / \mathrm{g}$, whereas the measured sensitivity of the ADXL50 of $20 \mathrm{mV} / \mathrm{g}$ is considerably higher than the predicted value. The difference is caused by signal attenuation by stray capacitances $(6 \mathrm{~dB})$, and by an additional amplifier stage adding $52 \mathrm{~dB}$ gain.

\section{Analysis of the performance}

To evaluate the performance, both dynamic range and frequency range should be taken into account. From the expression that Kühnel and Sherman give for the sensitivity, it can directly be seen that a trade-off has to be made concerning sensitivity and resonance frequency. The product of the sensitivity and the resonance frequency squared is the electrical field strength in the air gap. The amplifier output voltage swing and the air-gap thickness are thus key parameters for optimising the sensitivity and bandwidth of the accelerometer. The design trade-off between the sensitivity and bandwidth can be seen from the data listed in Table 2 . The ADXL50 has a high resonance frequency of $20 \mathrm{kHz}$, and consequently a low sensitivity. In contrast, the A-SMAC shows a high sensitivity of $5 \mathrm{~V} / \mathrm{g}$, which is needed for the desired $\mu \mathrm{g}$ resolution, at the cost of a low resonance frequency of $126 \mathrm{~Hz}$.

We will investigate with a design example if an accelerometer for modal analysis can be based on capacitive detection in combination with a feedback system. If we assume that the output voltage swing is limited to $+/-2 \mathrm{~V}$, and the desired working range $100 \mathrm{~m} / \mathrm{s}^{2}$, then the maximum sensitivity of the accelerometer becomes $20 \mathrm{mV} / \mathrm{m} / \mathrm{s}^{2}$. If the preamplifier noise level is $3 \mu \mathrm{V} / \sqrt{\mathrm{Hz}}$, this corresponds to a resolution of $1.5 \cdot 10^{4} \mathrm{~m} / \mathrm{s}^{2} / \sqrt{\mathrm{Hz}}$. If the desired resonance frequency is 5 $\mathrm{kHz}$, and the polarisation voltage $V_{p}$ is $2 \mathrm{~V}$, equation (36.) gives an air-gap thickness of $0.10 \mu \mathrm{m}$. This is a value which is technologically very hard to realise, and has to our knowledge not been demonstrated in any of the published silicon accelerometers. The noise level can be increased with a factor of 7 , to $1 \cdot 10^{3} \mathrm{~m} / \mathrm{s}^{2} / \sqrt{\mathrm{Hz}}$. This allows the air-gap thickness to be increased to $0.7 \mu \mathrm{m}$, which is still a factor of $3 \mathrm{~s}$ maller than the smallest practically used values in accelerometer constructions fabricated by wafer bonding techniques. As can be seen from equation (36.), the limiting factor for improvement of the sensitivity - bandwidth product is the electrical field strength. So either the airgap can be made smaller, or the electronics should be adjusted so that the used polarisation voltage can be increased. Apart from possible complications with increasing the voltages in the electronics with a factor 3 , other problems of an increased elec trical field strength in the airgap have to be considered, such as the risk for electrical discharge, and electrostatic collapse, 
where the seismic mass gets unstable and is attracted to one of the electrodes.

Recent developments with capacitive force-balanced accelerometers have resulted in completely digital devices (e.g. ${ }^{(26),(27)}$ ), where a sigma-delta converter detects if the position of the seismic mass differs from its rest position, and gives a feedback signal as a series of voltage pulses, which at the same time is the bitstream output of the accelerometer. This integrates the $\mathrm{AD}$ conversion with the accelerometer electronics. Other digital techniques have been presented, as for instance Pulse Width Modulation feedback (PWM) by Suzuki et al. ${ }^{(23)}$. We will not further analyse the performance of the digital accelerometers, but we believe the physical limitations to the performance are the same as in the analog accelerometers (buffer amplifier noise, accelerometer sensitivity and the electrostatic forces in the feedback system).

Summarising, capacitive force-balanced accelerometers have been demonstrated with low detection levels and a dynamic range of $100 \mathrm{~dB}$. However, the sensitivity-bandwidth product of these accelerometers is limited, which makes them less suitable for modal analysis applications where both high sensitivity and bandwidth are required.

<4.3> Electron tunnelling detection In 1989, Waltman and Kaiser ${ }^{\left({ }^{*}\right)}$ from Jet Propulsion Laboratories presented the first electron tunnelling accelerometer. This accelerometer was machined in aluminium, and the tunnelling current between two gold electrodes was controlled with a piezoelectric cantilever. To sustain a tunnelling current, the two gold electrodes have to brought at a distance of typically $10 \AA$. The used tunnelling current is typically around $1 \mathrm{nA}$, and depends exponentially on the electrode separation:

$$
I_{t}=I_{0} e^{-\frac{x}{x_{0}}}
$$

where $I_{0}$ is a function of the applied voltage between the conductors and

$$
x_{0}=\frac{1}{\alpha \sqrt{\phi}}
$$

where $\alpha=1.025 \cdot 10^{10} \mathrm{~m}^{-1} \mathrm{eV}^{-1 / 2}$ and $\phi$ is the effective work function of the surfaces. For gold to gold tunnelling, $\phi$ is about $0.5 \mathrm{eV}$ and $x_{0}$ becomes approximately $1 \AA$. The tunnelling current is a strongly nonlinear function of the distance $x$. For each Angström displacement, the current changes by nearly a factor of three. The sensitivity of a tunnelling accelerometer is the derivative of equation (37.):

$$
-\frac{\partial I}{\partial x}=\frac{I_{t}}{x_{0}} \quad \ldots . . . . . . .(39)
$$

With $I_{t}=1.3 \mathrm{nA}$ and $x_{0}=1 \AA$, the sensitivity becomes 9.4 $\mathrm{A} / \mathrm{m}$.

Noise level, dynamic range and Bandwidth

Due to the high sensitivity of the tunnelling accelerometer, very low accelerations can be detected even when an amplifier with a normal noise performance $\left(10^{-14}-10^{-15} \mathrm{~A} / \sqrt{\mathrm{Hz}}\right)$ is used. In practice, the amplifier noise may tum out to be less relevant than other noise sources, as for instance the shot noise in the tunnelling junction, or the mechanical-thermal noise level of the accelerometer. The shot noise current, is given by equation (19.). For a tunnelling current and gap given above the shot noise becomes $2 \cdot 10^{-14} \mathrm{~A} / \sqrt{ } \mathrm{Hz}$. This corre sponds to a displacement noise of $2 \cdot 10^{-15} \AA / \sqrt{ } \mathrm{Hz}$.

The upper detection limit is set by the feedback system. If a similar electrode configuration is used as with the capacitive feedback accelerometer, the same expression as in the previous section can be used for calculating the working range.

The influence of the three noise sources is shown in a design example, of which the data are shown in Table 3 .

\begin{tabular}{|l|l|}
\hline Seismic mass & $1 \cdot 10^{7} \mathrm{~kg}$ \\
\hline Spring constant & $100 \mathrm{~N} / \mathrm{m}$ \\
\hline Resonance frequency & $5 \mathrm{kHz}$ \\
\hline Air gap thickness & $5 \mu \mathrm{m}$ \\
\hline Electrode area & $3.5 \mathrm{~mm}^{2}$ \\
\hline Max. feedback voltage & $2 \mathrm{~V}$ \\
\hline Acceleration sensitivity & $9.4 \cdot 10^{-9} \mathrm{~A} / \mathrm{m} / \mathrm{s}^{2}$ \\
\hline Working range & $100 \mathrm{~m} / \mathrm{s}^{2}$ \\
\hline Noise level: & \\
\hline Thermal $(\mathrm{Q}=20$ assumed) & $1.6 \cdot 10^{-5} \mathrm{~m} / \mathrm{s}^{2} / \sqrt{ } \mathrm{Hz}$ \\
\hline HzShot noise & $2.3 \cdot 10^{-6} \mathrm{~m} / \mathrm{s}^{2} / \sqrt{\mathrm{Hz}}$ \\
\hline Preamplifier & $1.1 \cdot 10^{-6} \mathrm{~m} / \mathrm{s}^{2} / \sqrt{\mathrm{Hz}}$ \\
\hline
\end{tabular}

Table 3. Tunnelling accelerometer design example.

The example above shows that, due to the high sensitivity, the mechanical-thermal noise determines the noise level, rather than shot noise or preamplifier noise. Note that the shot noise was based on a current of $1.5 \mathrm{nA}$, and the assumed preamplifier noise was $1 \cdot 10^{14} \mathrm{~A} / \sqrt{ } \mathrm{H} z$, which is a conservative value. Although this is just a simple example, it shows that the preamplifier noise is lower than the contributions of other noise sources. Therefore, researchers at JPL and Stanford University working with tunnelling sensors use simple opamp-based electronics, and do not focus on noise optimisation of the amplifiers ${ }^{\text {in? }}$.

Until now, the work from the researchers at JPL and Stanford University on accelerometers was primarily focused on devices with a limited frequency range $(100 \mathrm{~Hz})$, but with a detection level lower than $10^{8} \mathrm{~g} / \sqrt{ } \mathrm{Hz}{ }^{(3 !)}$. A drawing of such an accelerometer is shown in figure 5 . Using a $100 \mathrm{mg}$ seismic mass and resonance frequency of $100 \mathrm{~Hz}$, a noise level of $1.5 \cdot 10^{7} \mathrm{~g} / \sqrt{ } \mathrm{Hz}$, at a frequency of $10 \mathrm{~Hz}$, were obtained. The accelerometers sensitivity was $15 \mathrm{kV} / \mathrm{g}^{(31)}$. 


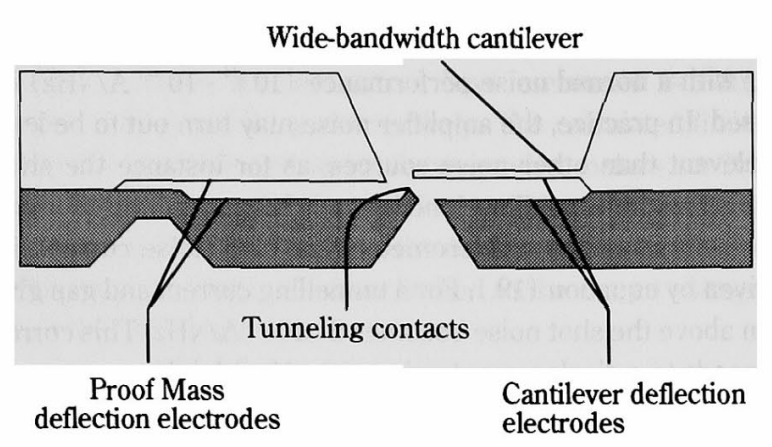

Fig. 5. Tunnelling accelerometer, after Rockstadt et al. ${ }^{(31)}$.

Research on electron tunnelling accelerometers started only a few years ago, and electron tunnelling based sensors are not yet on the market. However the results presented up to now look promising. An infrared tunnelling sensor has been reported to operate continuously for more than 18 months (by the time the publication was written), Kenny ${ }^{(30)}$. Many subjects are still under investigation, as for example the influence of tunnel electrode cleanliness (important for longterm stability, $\left.{ }^{(30)}\right)$, noise performance ${ }^{(32)}$, and the feedback control system ${ }^{(33)}$. From the design example given above, the expected dynamic range, noise level and bandwidth are sufficient for modal applications.

\section{Discussion}

Piezoresistive and piezoelectric accelerometers exhibit similar dynamic ranges (both approximately $130 \mathrm{~dB}$ ), in combination with a sufficient frequency range for modal applications. The piezoresistive accelerometers are produced today, whereas piezoelectric accelerometers only have been presented in a few publications. Since the piezoresistors only have to cover a small area to sense strain, piezoresistive accelerometers can easily be scaled down to dimensions suitable for silicon micromachining, whereas scaling piezoelectric accelerometers is limited, since piezoelectric strain gauges have to cover a certain area, needed for generating sufficient charge for the charge amplifier to measure.

The Bragg grating strain detection will probably find special application areas due to the inherent immunity to EMI and other disturbances, but much work remains to be done, also on the demodulation technique.

Scaling resonant strain gauges is inherent to the detection principle, since resonator frequencies of the order of $100 \mathrm{kHz}$ are desired for operation. We believe the applications of resonant accelerometers are limited to applications at low frequencies, such as inertial navigation. With a limited bandwidth, the resonant accelerometer shows a high dynamic range and resolution.

The capacitive feedback technology has reached the same state as the piezoresistive technology, and a number of these accelerometers is commercially available today. With capacitive feedback accelerometers, the sensitivity-bandwidth prod- uct is limited by the electrical field strength in the airgap. The commercially available accelerometers therefore show either a high resolution and dynamic range in a limited bandwidth, as for example the CSEM accelerometer for inertial navigation, or a low resolution in an extended bandwidth, as for instance the $\mathrm{ADXL} 50$ for airbag applications. We believe that the limited sensitivity-bandwidth product makes these accelerometers less suitable for modal and more general purpose type applications.

Electron tunnelling accelerometers are very well suitable to downscaling, since the active area for sensing is a very small tunnelling tip, thus not having scaling restrictions to the area as with piezoelectric or capacitive accelerometers. Tunnelling accelerometers have been demonstrated with noise levels less than $0.1 \mu \mathrm{g}$ in a $100 \mathrm{~Hz}$ bandwidth. We expect that these accelerometers can show a low noise level in combination with a high bandwidth. However, issues as long-term stability and shock protection of these accelerometers still have to be clarified. The next decade will show if these sensors find their way to the market.

In the 17 years after the first micromachined silicon accelerometer from Roylance and Angell, silicon accelerometers based on many different transduction techniques have been developed, and some found their way to the market. Silicon micromachining allowed downscaling of dimensions, thus making massfabrication of reliable and low cost accelerometers for airbag applications possible. The high precision and new possibilities of the micromachining technology resulted in new types of accelerometers, as for instance the resonant accelerometer and the force-balance capacitive accelerometer. These accelerometers can further increase reliability, for example by the continuous self-test of a resonant accelerometer.

To take advantage of the potentially low price of the accelerometer chip new more cost effective methods of encapsulation and connection have to be developed if the accelerometers shall be suitable for the general purpose market of today.

New technologies are being investigated, such as the electron tunnelling accelerometer, and the optical accelerometers. The new transduction principles will lead to new limits for miniaturisation and noise level. Some of the new transduction principles will make new accelerometers find their way to the market in the next decade, but a revolution as in the computer world does not seem imminent.

(Manuscript received August 7, '96)

\section{References}

(1) Lynn Michelle Roylance, James B. Angell, "A Batch-Fabricated Silicon Accelerometer", IEEE Tran.Elec.Dev, 1979, pp.1911-1917.

(2) Bo-Shen Zhu, Mette Owner-Petersen and Torben Licht, "Accelerometer design based on attenuated total reflection", Applied Optics, Vol.27, No.14, 1988, pp. 2972-2975.

(3) Smith, F.E. Jones and R.P. Chasmar,"The detection and 
measurement of infra-red radiation", Oxford University Press 1968.

(4) Torben Storgaard-Larsen et al,"Opto-mechanical accelerometer based on strain sensing by a Bragg grating in a planar waveguide", Sensors and Actuators, A52, 1996, pp.25-32.

(5) Bouwstra, "Resonating mocrobridge mass flow sensor",PhD Thesis, University of Twente, 1990.

(6) L.A.Christel et al."Vibration Rectification in Silicon Micromachined Accelerometers”, transducer '91, San Francisco 1991, pp.88-92.

(7) H.Crazzolara, G.Flach and W.von Münch,"Piezoresistive accelerometer with overload protection and low crosssensitivity", Sensors and Actuators A, 1993, pp.201-207.

(8) S.C.Terry et al."Self-Testable Accelerometer Microsystem", Micro System technologies 90, Sprinegr, Berlin, 1990, pp.611-616.

(9) Anton Heuberger, "Mikromechanik", Springer Verlag, 1989, pp. 366-380.

(10) Jarnes T. Suminto, Endevco,"A Wide frequency range, Rugged Silicon micro Accelerometer with Overrange stops", IEEE MEMS96, 1996, pp. 180-185.

(11) P.W.Barth et al,"A Monolithic Silicon Accelerometer with integral air damping and overrange shock protection", Tech. Dig., IEEE Solid State Sensor and Actuator, Hilton Head 1988, pp.35-38.

(12) H.V.Allen et al."Accelerometer Systems with SelfTestable Features", Sensors and Actuators, Vol.20, 1989, pp.153-161.

(13) Thomas Tschan,"Simulation, Design and Characterization of a Silicon Piezoresistive Accelerometer, Fabricated by a Bipolar-Compatible Industrial Process", PhD Dissertation, University of Neuchâtel, 1991.

(14) J.Bryzek et al."New Technologies for Silicon Accelerometers Enable Automotive Applications", Proc. of the SAE International Congress and Exposition, Detroit MI, 1992, pp.25.

(15) P.L.Chen et al."Integrated Silicon Microbeam PI-FET Accelerometer", IEEE Trans. Electron Devices, Vol. FD-29, 1982, pp.27-33.

(16) Patrick Sheeper et al.,"A piezoelectric triaxial accelerometer", J. Micromech. Microeng. 6 (1996) pp. 131-133.

(17) Berlincourt, "Ultrasonic technologi ", Plenum Press 1971, pp.63-124.

(18) C.P. Germano,"Flexure mode piezoelectric transducers", IEEE Trans. on Audio and Electroacoustics, Vol.
AU-19, No.1, 1971, pp.6-12.

(19) Terry V. Roszhart, Hal Jerman et al, I.C. Sensors,"An Inertial Grade Micromachined Vibrating Beam Accelerometer", Transducers '95, Vol.2, pp.656-659.

(20) H. Jakobsen, "Sensor foundries and Production of sensors at Sensor A.S.", Technical Digest, MME '95.

(21) SensoNor A.S., Horten, Norway, "Datasheet for SA30 Crash Sensor".

(22) D.W.Burns et al, Honeywell Tech,"Resonant Microbeam Accelerometers", Transducers '95, Vol.2, pp.659-662.

(23) Wolfgang Kuehnel and Steven Sherman,"A surface micromachined silicon accelerometer with on-chip detection circuitry", Sensors and Actuators A45, 1994, pp.716.

(24) F.Rudolf et al, CSEM, "Precision Accelerometers with $\mu \mathrm{g}$ Resolution", Sensors and Actuators A21-23, 1990, pp.297-302.

(25) H.Leuthold and F. Rudolf, CSEM,"An ASIC for Highresolution Capacitive Microaccelerometers", Sensors and Actuators A21-23, 1990, pp.278-281.

(26) W.Henrion, Hal Jerman et al, 1.C. Sensors. "Wide dynamic range direct digital accelerometer", IEEE Hilton Head 1990, pp.153-157.

(27) Y.de Coulon et al, CSEM,"Design and test of a precision servoaccelerometer with digital output", 7th Solid State Sensors and Actuators, 1993, pp. 832-835.

(28) S.Suzuki and S.Tuchitani,"Semiconductor Capacitancetype Accelerometer with PWM Electrostatic Servo technique", Sensors and Actuators, Vol. A21-23, 19990, pp.316-319.

(29) Waltman and W.J. Kaiser, "An electron tunneling sensor", Sensors and Actuators, vol.. 19, 1989, 201-210.

(30) T.W.Kenny et al."Wide-Bandwith Electromechanical Actuators for Tunneling Displacement Transducers", J.Microelectromechanical Systems, Vol.3, No.3, September 1994, pp.97-104.

(31) Howard K. Rockstadt, T.W.Kenny et al."A Miniature, High-Sensitive, Electron Tunneling Accelerometer", Transducers '95, Vol.2, pp.675-678.

(32) J.Grade et al."Progress in tunnel Sensors", Preprint Hilton Head 1996.

(33) Liu, J.K. Reynolds, A Partridge, T.W. Kenny, L.M. Miller, J.A. Podosek, H.K. Rockstad, W.J. Kaiser, "High-bandwidth feedback control of micromachined tunneling sensors", Proceedings ASME Dynamic Systems and Control Division, 1995, pp. 1001-1010. 